\title{
Long noncoding RNA PURPL promotes cell proliferation in liver cancer by regulating p53
}

\author{
XUEYAN FU ${ }^{1}$, YAWEI WANG ${ }^{2}$, GANG WU $^{1}$, WANCHUAN ZHANG ${ }^{1}$, SHAOLIN XU ${ }^{1}$ and WENQING WANG ${ }^{1}$ \\ Departments of ${ }^{1}$ General Surgery and ${ }^{2}$ Geriatric Surgery, \\ The First Affiliated Hospital of China Medical University, Shenyang, Liaoning 110001, P.R. China
}

Received August 1, 2018; Accepted April 5, 2019

DOI: $10.3892 / \mathrm{mmr} .2019 .10159$

\begin{abstract}
Emerging evidence suggests that long noncoding RNAs (lncRNAs) serve a key role in malignant transformation, tumor progression and metastasis. Increased expression of lncRNA p53 upregulated regulator of P53 levels (PURPL) has been reported to promote tumorigenicity in colorectal cancer; however, the role and potential mechanisms of PURPL in the development of liver cancer remain unclear. We employed reverse transcription-quantitative polymerase chain reaction to detect PURPL and p53 mRNA expression in liver cancer tissues and cell lines. Cell Counting Kit-8 and colony-forming assays were used to examine the cell proliferation; whereas, flow cytometry was applied to detect apoptosis and cell cycle progression. p53 expression was detected by western blotting. The results revealed that PURPL was significantly upregulated in liver cancer tissues compared with in paracancerous tissues, and was associated with tumor differentiation stage and tumor size. PURPL was also upregulated in various liver cancer cell lines. Silencing of PURPL inhibited liver cancer cells proliferation, blocked cell cycle progression, and promoted apoptosis. Most importantly, PURPL expression was negatively correlated with p53 mRNA expression. In summary, lncRNA-PURPL was proposed to promote cell proliferation in liver cancer by regulating the p53 gene. As such, it could serve as a potential therapeutic target for the diagnosis and treatment of liver cancer.
\end{abstract}

\section{Introduction}

Liver cancer is the sixth most common cancer and the third most common cause of cancer-associated mortality worldwide $(1,2)$. Liver resection remains the best therapeutic strategy to treat liver cancer, with a 5-year survival rate

Correspondence to: $\mathrm{Dr}$ Gang Wu, Department of General Surgery, The First Affiliated Hospital of China Medical University, 155 Nanjingbei Street, Shenyang, Liaoning 110001, P.R. China E-mail: cmuwgzwl@126.com

Key words: apoptosis, cell cycle, liver cancer, long noncoding RNA-p53 upregulated regulator of P53 levels, p53, proliferation of $\sim 30 \%$. Chronic infection with hepatitis $\mathrm{B}$ or hepatitis $\mathrm{C}$ virus is the most common cause of liver cancer (3); however, the mechanism underlying the development of the disease remain unclear. Advancements in molecular technology have led to a focus on the molecular mechanism of liver cancer progression, and the identification of potential clinically relevant factors which may benefit the diagnosis and treatment of liver cancer.

Long non-coding RNAs (lncRNAs) are long-chain RNA molecules of $>200$ nucleotides in length that do not encode for proteins; thus, they have been proposed to be ineffective (4). Numerous studies have reported that lncRNAs are closely related to the multi-level regulation of gene expression, particularly the post-transcriptional regulation of the interaction with microRNAs (miRNAs), mRNAs, or proteins (5-8). Recent evidence suggests that lncRNAs are involved in several pathological processes driving cancer, including proliferation, apoptosis, metastasis and metabolism (9-13). In particular, certain IncRNAs have been reported to be critical in the progression of liver cancer. LncRNA p53 upregulated regulator of P53 levels (lncRNA-PURPL), an intergenic lncRNA, has been identified by RNA sequencing in a variety of colorectal cancer cell lines. It has been observed to promote the occurrence of colorectal cancer by preventing the binding of p53 to the small p53-stabilizing protein Myb-binding protein 1A (MYBBP1A), lowering p53 levels (14). However, to the best of our knowledge, no investigations into the role and expression of PURPL in liver cancer have been conducted.

The present study reported that PURPL is significantly upregulated in liver cancer tissues and cell lines. We also demonstrated that PURPL could promote liver cancer cell proliferation and cell cycle progression, while inhibiting apoptosis via regulation of the p53 gene. These findings may provide novel insight into the role of PURPL in the progression of liver cancer.

\section{Materials and methods}

Tissue collection and cell culture. Primary tumor tissue and corresponding adjacent tissue were excised from 71 patients (60 males and 11 female; age range: 30-80-years-old) at The First Affiliated Hospital of China Medical University between December 2016 and May 2018. The isolated tissue specimens were placed in a cryotube within $30 \mathrm{~min}$ and 
immediately frozen in a $-80^{\circ} \mathrm{C}$ freezer. All cancerous tissues were pathologically diagnosed as liver cancer. Patients did not receive preoperative radiotherapy or chemotherapy. All individuals provided informed consent and the present study was approved by the Institutional Ethics Committee of China Medical University. Patients were divided into high- and low-PURPL expression groups based on the median value of expression.

A total of five liver cancer cell lines, HepG2, Huh7, HCCLM3, SK-hep1, and PLC/PRF/5, as well as the normal liver cell line, L02, were used in this study. HepG2 cells were obtained from the Chinese Academy of Medical Sciences (Beijing, China). Huh7, SK-hep1, PLC/PRF/5, HCCLM3 and L02 human cell lines were obtained from the Chinese Academy of Sciences (Shanghai, China). SK-hep1 and L02 cells were cultured in RPMI 1640 medium (HyClone; GE Healthcare, Logan, UT, USA). HepG2, Huh7, PLC/PRF/5 and HCCLM3 cells were cultured in Dulbecco's Modified Eagle's medium (Gibco; Thermo Fisher Scientific, Inc., Waltham, MA, USA). Media were supplemented with $10 \%$ fetal bovine serum and $100 \mathrm{U} / \mathrm{ml}$ penicillin and $100 \mathrm{U} / \mathrm{ml}$ streptomycin (HyClone; GE Healthcare). All cells were grown at $37^{\circ} \mathrm{C}$ in a humidified incubator containing $5 \% \mathrm{CO}_{2}$.

Small interfering RNA (siRNA) and transfection. A total of three siRNAs for the silencing of PURPL and a negative control siRNA (si-NC) were designed and synthesized by Shanghai GenePharma Co., Ltd. (Suzhou, China). Cell lines cultured in six-well plates were transfected with $20 \mu \mathrm{M}$ siRNAs dissolved in $\mathrm{dd}_{2} \mathrm{O}$ using Lipofectamine ${ }^{\circledR} 2000$ (Invitrogen; Thermo Fisher Scientific, Inc.) and mixed with siRNA-PURPL or si-NC according to the manufacturer's protocols. After $48 \mathrm{~h}$, cells were collected for reverse transcription-quantitative polymerase chain reaction (RT-qPCR), western blotting and other experiments. The sequences of the siRNAs were as follows: 1\#PURPLsiRNA, 5'-CCACAUACAGGGUUCUUAATT UUAAGA ACCCUGUAUGUGGTT-3'; 2\#PURPLsiRNA, 5'-GGAAUCGAUCUGUGAGCAUTTAUGCUCACAGAU CGAUUCCTT-3'; 3\#PURPLsiRNA, 5'-GGCCUACGUGAA UAAUAAUTTAUUAUUAUUCACGUAGGCCTT-3'; and si-NC sense, 5'-UUCUCCGAACGUGUCACG-3' and si-NC antisense, 5'-ACGUGACACGUUCGGAGAATT-3'. Among them, 3\#PURPLsiRNA with the strongest silencing efficiency, was used in subsequent experiments.

$R N A$ extraction and $R T-q P C R$. Total RNA was extracted from clinical patient tissue samples and cultured cells using Total RNA Extractor (Sangon Biotech Co., Ltd., Shanghai, China) according to the manufacturer's instructions. Total RNA was reverse transcribed into cDNA using the GoScript ${ }^{\mathrm{TM}}$ Reverse Transcription Mix and Random Primers (Promega Corporation, Madison, WI, USA) according to the the manufacturer's instructions. The cDNA was then amplified by qPCR using the GoTaqR qPCR Master Mix (Promega Corporation) according to the manufacturer's instructions on an ABI PRISMR 7500 (Applied Biosystems; Thermo Fisher Scientific, Inc.) instrument using a two-step standard PCR amplification procedure. The first step (pre-denaturation) included a cycle of $2 \mathrm{~min}$ at $95^{\circ} \mathrm{C}$; the second step (PCR reaction) included 40 cycles of $15 \mathrm{sec}$ at $95^{\circ} \mathrm{C}$ and $1 \mathrm{~min}$ at $60^{\circ} \mathrm{C}$. Finally, the relative expression of PURPL and p53 mRNA in tissue and cell samples was determined by the $2^{-\Delta \Delta \mathrm{Cq}}$ method (15). GAPDH was used as a control. The nucleotide sequences of the primers for qPCR were as follows: PURPL forward, 5'-TTCTACCGC AATTCGATGGAGTCTTG-3', reverse, 5'-GAGGCAGGA GAATGGCGTGAAC-3'; p53 forward, 5'-ACCGGCGCACAG AGGAAGAG-3', reverse, 5'-GCCTCATTCAGCTCTCGG AACATC-3'; and GAPDH forward, 5'-CGGAGTTGTTCG TATTCGG-3' and reverse, 5'-TACTAGCCGATGATGGC ATT-3'.

Western blotting. Transfected cells and frozen liver tissues, including liver cancer and corresponding adjacent tissues, were subjected to western blotting to evaluate p53 protein content. Cells and tissue samples were lysed for $30 \mathrm{~min}$ in radioimmunoprecipitation assay buffer (Beyotime Institute of Biotechnology, Shanghai, China) on ice, the resulting lysates were centrifuged at $10,000 \mathrm{x}$ g for $15 \mathrm{~min}$ at $4^{\circ} \mathrm{C}$, and the protein concentration was measured using a Bicinchoninic Acid kit (FDbio Science, Hangzhou, China). Protein samples $(30 \mu \mathrm{g})$ were then separated by conventional electrophoresis at $70 \mathrm{~V}$, transferred under constant pressure, blocked in 5\% skim milk, and finally incubated with anti-p53 (cat. no. 60283-l-lg, 1:1,000) or anti-GAPDH (cat. no. 60004-1-lg, $1 \mathrm{mg} / \mathrm{ml}$ ), ProteinTech Group, Chicago, IL, USA) primary antibodies at $4^{\circ} \mathrm{C}$ overnight. The following day, membranes were washed with PBS, incubated with a secondary antibody (Goat anti-mouse IgG, horseradish peroxidase, cat. no. ZB-2305, 1:10,000, ZSGB-BIO) for $1 \mathrm{~h}$ at room temperature, and proteins were visualized by chemiluminescence using an ECL kit and an ECL western blotting substrate (Pierce; Thermo Fisher Scientific, Inc.). Densitometry analysis was performed using ImageJ software version 1.8.0 (National Institutes of Health, Bethesda, MD, USA).

Cell proliferation assays. Transfected HepG2 and Huh7 cells were seeded in 96-well microtiter plates at a density of $5 \times 10^{3}$ cells/well. At 24, 48, 72 and $96 \mathrm{~h}$ post-transfection, $10 \mu \mathrm{l}$ Cell Counting Kit-8 (CCK-8) solution (Beyotime Institute of Biotechnology) was added to each well and the plates were incubated in a $37^{\circ} \mathrm{C}$ incubator for $1.5 \mathrm{~h}$. Then, the absorbance per well was measured at $450 \mathrm{~nm}$ using an automatic microplate reader (BGI, Hong Kong, China).

For the colony-forming assay, each group of cells was seeded on a six-well plate at $1 \times 10^{3}$ cells/well and incubated for 10 days at $37^{\circ} \mathrm{C}$ in $5 \% \mathrm{CO}_{2}$. Then, the cells were washed with PBS, fixed with paraformaldehyde for $15 \mathrm{~min}$ then stained with $0.5 \%$ crystal violet at room temperature and colonies were then counted.

Cell cycle analysis. To investigate the effects of PURPL silencing on cell cycle progression, we analyzed the proportion of cells in G1, S, and G2 phase using a BD FACSCanto ${ }^{\mathrm{TM}}$ flow cytometer (BD Biosciences, Franklin Lakes, NJ, USA). We routinely detached cells with trypsin $(0.25 \%)$ for $5 \mathrm{~min}$ at $37^{\circ} \mathrm{C}$, centrifuged at $850 \mathrm{x} \mathrm{g}$ at $4^{\circ} \mathrm{C}$ for $5 \mathrm{~min}$, removed the supernatant, resuspended the cells in pre-cooled PBS, and centrifuged them again. The supernatant was aspirated, and the cells were fixed at $4^{\circ} \mathrm{C}$ by adding $1 \mathrm{ml}$ of pre-cooled $70 \%$ ethanol and subsequent incubation for $12 \mathrm{~h}$. The next day, following 

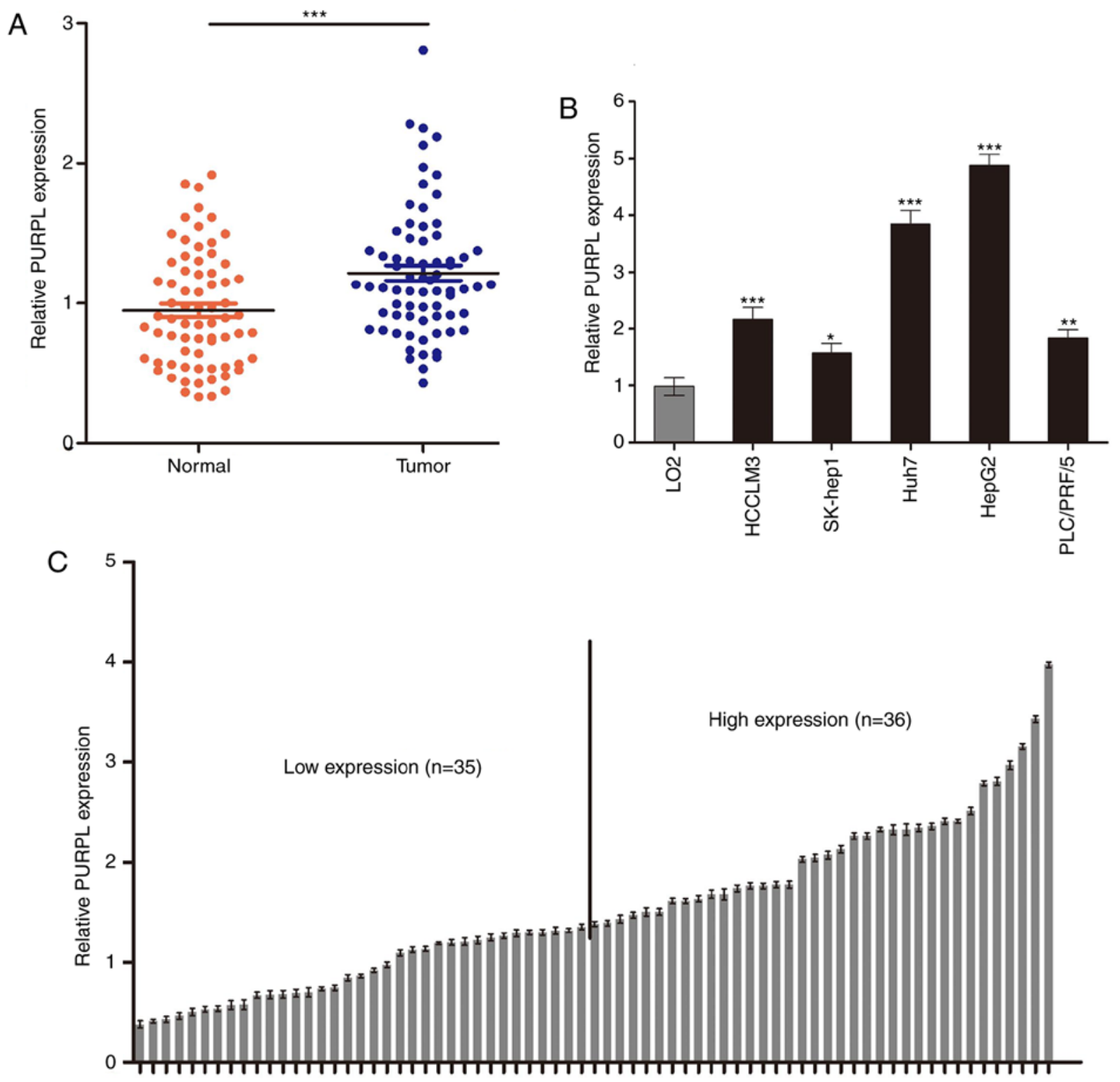

Liver cancer patients $(n=71)$

Figure 1. LncRNA-PURPL is upregulated in liver cancer tissues and cell lines. (A) Reverse transcription-quantitative polymerase chain reaction analysis of PURPL expression in 71 pairs of liver cancer and corresponding adjacent non-tumor control tissues. The results were analyzed using via a Student's t-test ${ }^{* * *} \mathrm{P}<0.001$. (B) Relative expression of PURPL in five liver cancer cell lines (HepG2, Huh7, HCCLM3, SK-hep1, PLC/PRF/5) and a normal liver cell line (L02). The results were analyzed using one-way analysis of variance followed by the Least Significant Difference test. (C) Division of the 71 patients with liver cancer into a high-expression PURPL group $(\mathrm{n}=36)$ and a low-expression PURPL group $(\mathrm{n}=35)$ based upon levels of median expression. ${ }^{*} \mathrm{P}<0.05,{ }^{* * *} \mathrm{P}<0.01$ and ${ }^{* * *} \mathrm{P}<0.001$ vs. L02. Error bars correspond to the standard deviation. PURPL, long noncoding RNA p53 upregulated regulator of P53 levels.

centrifugation $\left(850 \mathrm{x} \mathrm{g}, 4^{\circ} \mathrm{C}, 5 \mathrm{~min}\right)$ and washing(PBS), $0.5 \mathrm{ml}$ of propidium iodide (PI) staining solution (Beyotime Institute of Biotechnology) was added to each group of cell samples, which were incubated at $37^{\circ} \mathrm{C}$ for $30 \mathrm{~min}$ in the dark. Samples were then stored on ice until they were detected on the flow cytometer (BD FACSCanto ${ }^{\mathrm{TM}}$ ).

Cell apoptosis analysis. In accordance with the manufacturer's protocols of an apoptosis kit (Dojindo Molecular Technologies, Inc., Kumamoto, Japan), cells were trypsinized, collected $\left(\sim 5 \times 10^{5}\right.$ cells/group), washed twice with PBS, centrifuged at $850 \mathrm{x} \mathrm{g}$ at $4^{\circ} \mathrm{C}$ for $5 \mathrm{~min}$, and then $100 \mu \mathrm{l}$ of Annexin V buffer was added. Then, $5 \mu 1$ of PI and $5 \mu 1$ of Annexin V-FITC solution were added to each tube. The tubes were mixed by pipetting and incubated at room temperature for $15 \mathrm{~min}$, after which $400 \mu 1$ of Annexin V buffer (included in kit) was added to each tube and mixed by pipetting. Readings were obtained on a flow cytometer (BD FACSCanto ${ }^{\mathrm{TM}}$ ) as aforementioned.

Statistical analysis. The results were analyzed using GraphPad Prism 5.0 software (GraphPad Software, Inc., La Jolla, CA, USA). Data are expressed as the mean \pm standard deviation. Differences between two groups were assessed using a Student's t-test, whereas differences among multiple groups were analyzed using one-way analysis of variance followed by the Least Significant Difference test. The relationship between PURPL and p53 expression was analyzed by 
Table I. Association between lncRNA-PURPL expression according to reverse transcription-quantitative polymerase chain reaction and conventional clinicopathological parameters in 71 patients with liver cancer.

\begin{tabular}{|c|c|c|c|c|}
\hline Characteristics & $\begin{array}{c}\text { Number of } \\
\text { patients }\end{array}$ & $\begin{array}{c}\text { lncRNA-PURPL } \\
\text { Low expression }(\%)\end{array}$ & $\begin{array}{l}\text { IncRNA-PURPL } \\
\text { High expression }(\%)\end{array}$ & P-value \\
\hline Total cases & 71 & 35 & 36 & \\
\hline Age (years) & & & & 0.111 \\
\hline$\geq 55$ & 29 & $11(37.93)$ & $18(62.07)$ & \\
\hline$<55$ & 42 & $24(57.14)$ & $18(42.86)$ & \\
\hline Sex & & & & 0.705 \\
\hline Male & 60 & $29(48.33)$ & $31(51.67)$ & \\
\hline Female & 11 & $6(54.56)$ & $5(45.44)$ & \\
\hline Tumor size & & & & $0.013^{\mathrm{a}}$ \\
\hline$\geq 5 \mathrm{~cm}$ & 39 & $14(35.90)$ & $25(64.10)$ & \\
\hline$<5 \mathrm{~cm}$ & 32 & $21(65.63)$ & $11(34.37)$ & \\
\hline HBsAg status & & & & 0.591 \\
\hline Positive & 57 & $29(50.88)$ & $28(49.12)$ & \\
\hline Negative & 14 & $6(42.86)$ & $8(57.14)$ & \\
\hline Tumor differentiation & & & & $0.002^{\mathrm{b}}$ \\
\hline High & 21 & $17(80.95)$ & $4(19.05)$ & \\
\hline Moderate & 30 & $12(40.00)$ & $18(60.00)$ & \\
\hline Poor & 20 & $6(30.00)$ & $14(70.00)$ & \\
\hline PVTT & & & & 0.097 \\
\hline Yes & 10 & $2(20.00)$ & $8(80.00)$ & \\
\hline No & 61 & $33(54.10)$ & $28(45.90)$ & \\
\hline Serum AFP & & & & 0.288 \\
\hline$<20 \mathrm{ng} / \mathrm{ml}$ & 30 & $17(56.67)$ & $13(43.33)$ & \\
\hline$\geq 20 \mathrm{ng} / \mathrm{ml}$ & 41 & $18(43.90)$ & $23(56.10)$ & \\
\hline Liver cirrhosis & & & & 0.350 \\
\hline Yes & 56 & $26(46.43)$ & $30(53.57)$ & \\
\hline No & 15 & $9(60.00)$ & $6(40.00)$ & \\
\hline Metastasis & & & & 0.999 \\
\hline Yes & 7 & $3(42.86)$ & $4(57.14)$ & \\
\hline No & 64 & $32(50.00)$ & $32(50.00)$ & \\
\hline
\end{tabular}

${ }^{\mathrm{a}} \mathrm{P}<0.05,{ }^{\mathrm{b}} \mathrm{P}<0.01$.

Pearson correlation analysis. The association between PURPL expression and the clinicopathological parameters of patients was assessed via a $c^{2}$ test. All experiments were performed in triplicate. $\mathrm{P}<0.05$ was considered to indicate a statistically significant difference.

\section{Results}

LncRNA PURPL is upregulated in liver cancer tissues and cell lines. Using RT-qPCR, we examined the expression of PURPL in 71 patients with liver cancer and several liver cancer cell lines. The expression of PURPL was significantly increased in cancer tissues than in adjacent tissues $(\mathrm{P}<0.001$; Fig. 1A). In addition, PURPL was highly expressed in HepG2 and Huh7 cells compared with the three other liver cancer cell lines and L02 cells $(\mathrm{P}<0.05$; Fig. 1B). To investigate the association of PURPL expression with clinicopathological factors of patients, the patients were divided into high- $(\mathrm{n}=36)$ and low-expression $(n=35)$ groups based on PURPL median expression levels (Fig. 1C). As presented in Table I, PURPL expression levels in patients with liver cancer were closely associated with tumor size $(\mathrm{P}<0.05)$ and tumor differentiation $(\mathrm{P}<0.01)$. These data indicated that PURPL is significantly elevated in liver cancer patients and may be closely related to the progression of liver cancer.

Depletion of IncRNA PURPL suppresses liver cancer cell proliferation. To determine whether PURPL regulates the proliferative capacity of liver cancer cells, we used three different siRNAs to silence the PURPL gene in liver cancer cells. \#3PURPLsiRNA exhibited the strongest silencing efficiency ( $\mathrm{P}<0.05$; Fig. 2A and $\mathrm{B})$; thus, \#3PURPLsiRNA was selected for subsequent experiments. To determine the effect of PURPL on proliferation of liver cancer cells, we performed 

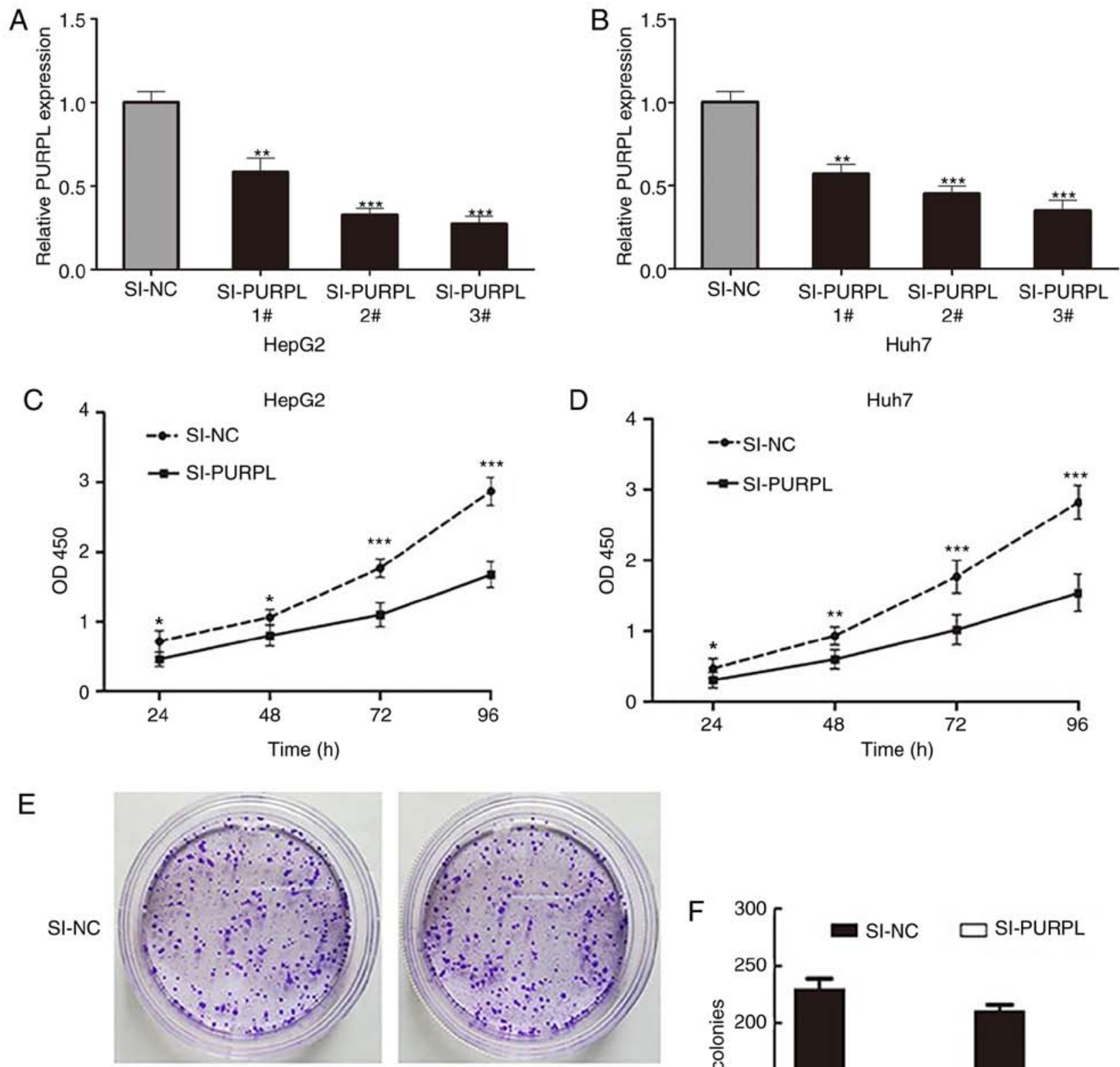

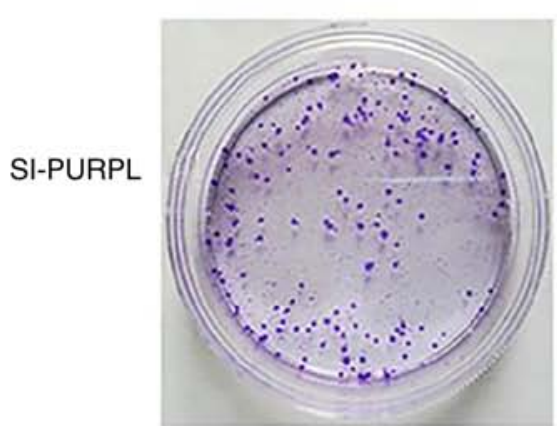

HepG2

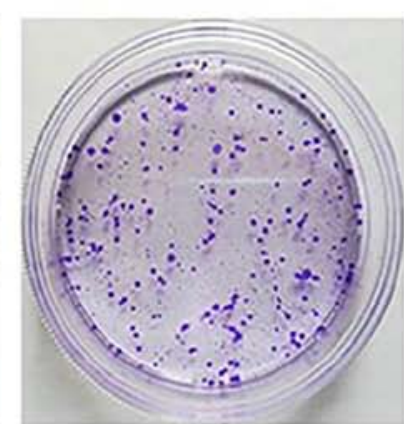

Huh7

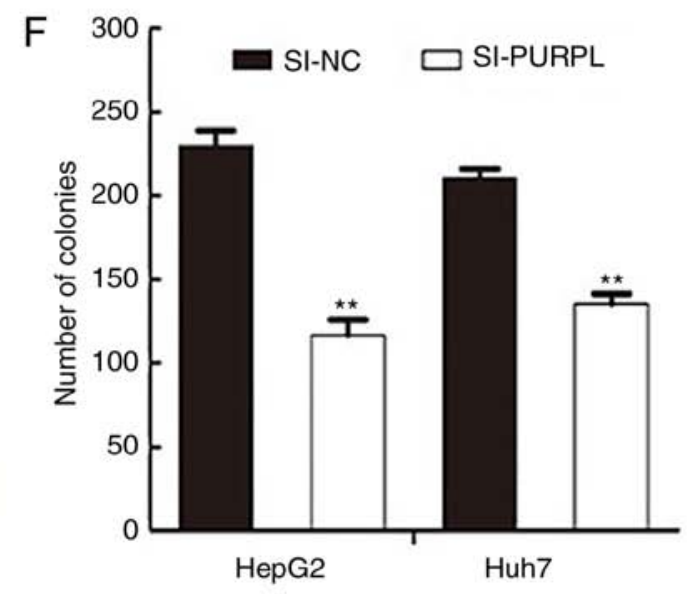

Figure 2. Long noncoding RNA-PURPL promotes the proliferation of HepG2 and Huh7 cells. (A and B) Efficiency of PURPL knockdown $24 \mathrm{~h}$ after transfecting cells with three SI-PURPL or SI-NC, as verified by reverse transcription-quantitative polymerase chain reaction. The results were analyzed using one-way analysis of variance followed by the Least Significant Difference test. (C and D) Effects of PURPL silencing on cell proliferation, as detected by a Cell Counting Kit-8 assay and absorbance measurements. (E and F) Effects of PURPL silencing on cell proliferation, as detected by colony-forming assays. The results were analyzed using a Student's t-test. ${ }^{*} \mathrm{P}<0.05,{ }^{* *} \mathrm{P}<0.01$ and $^{* * * *} \mathrm{P}<0.001$ vs. SI-NC. Error bars correspond to the standard deviation. NC, negative control; PURPL, p53 upregulated regulator of P53 levels; SI, small interfering RNA.

a CCK-8 assay. Silencing of PURPL significantly inhibited the proliferation of HepG2 and Huh7 cells compared with the control $(\mathrm{P}<0.05$; Fig. $2 \mathrm{C}$ and $\mathrm{D})$. In addition, the number of clones formed by PURPL-silenced cells was significantly decreased compared with the corresponding controls $(\mathrm{P}<0.01$; Fig. 2E and F). 
A
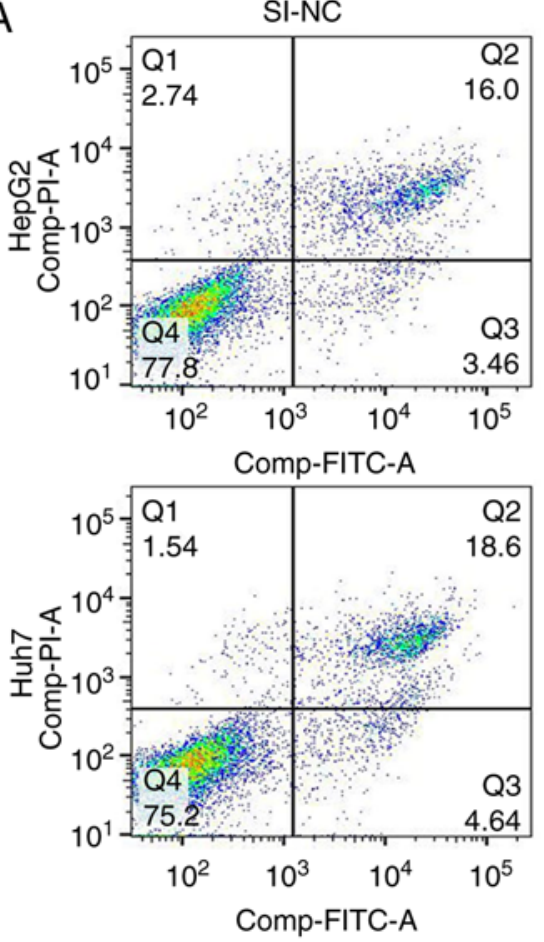

C
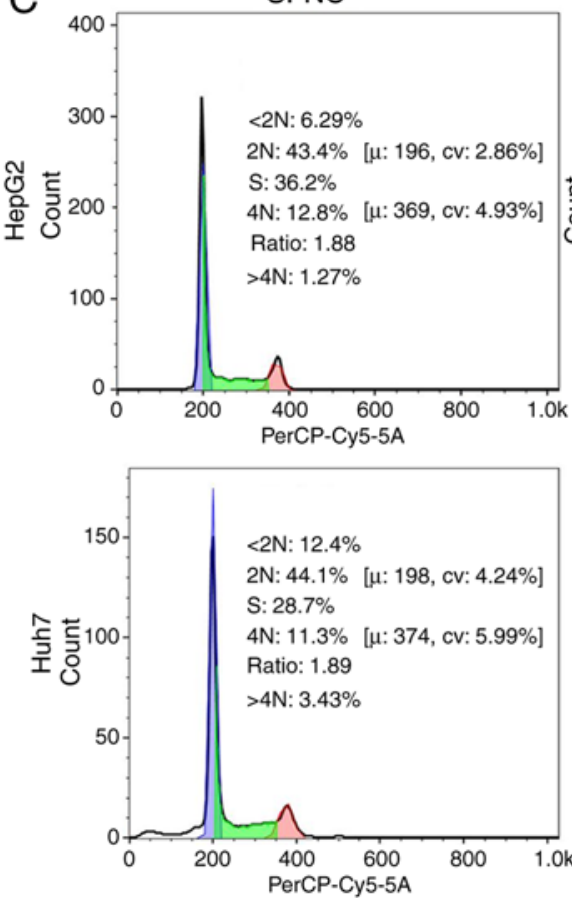

SI-PURPL
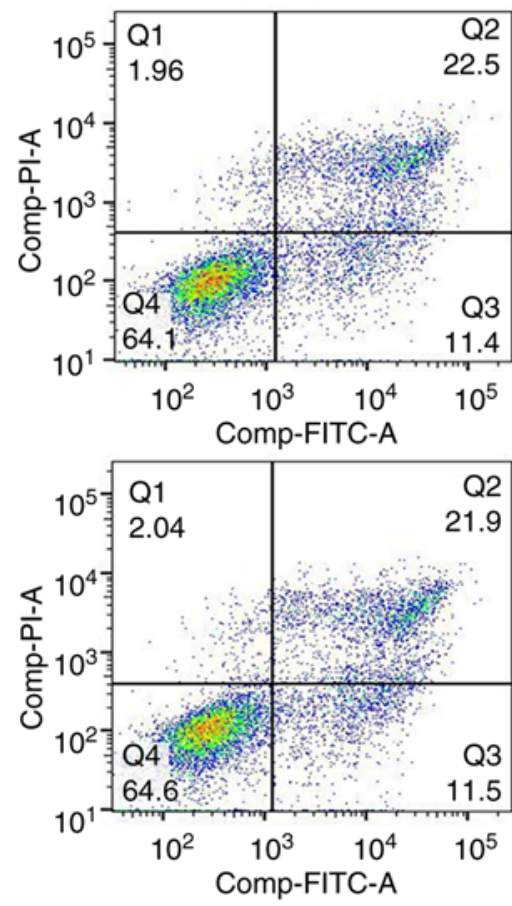

SI-PURPL
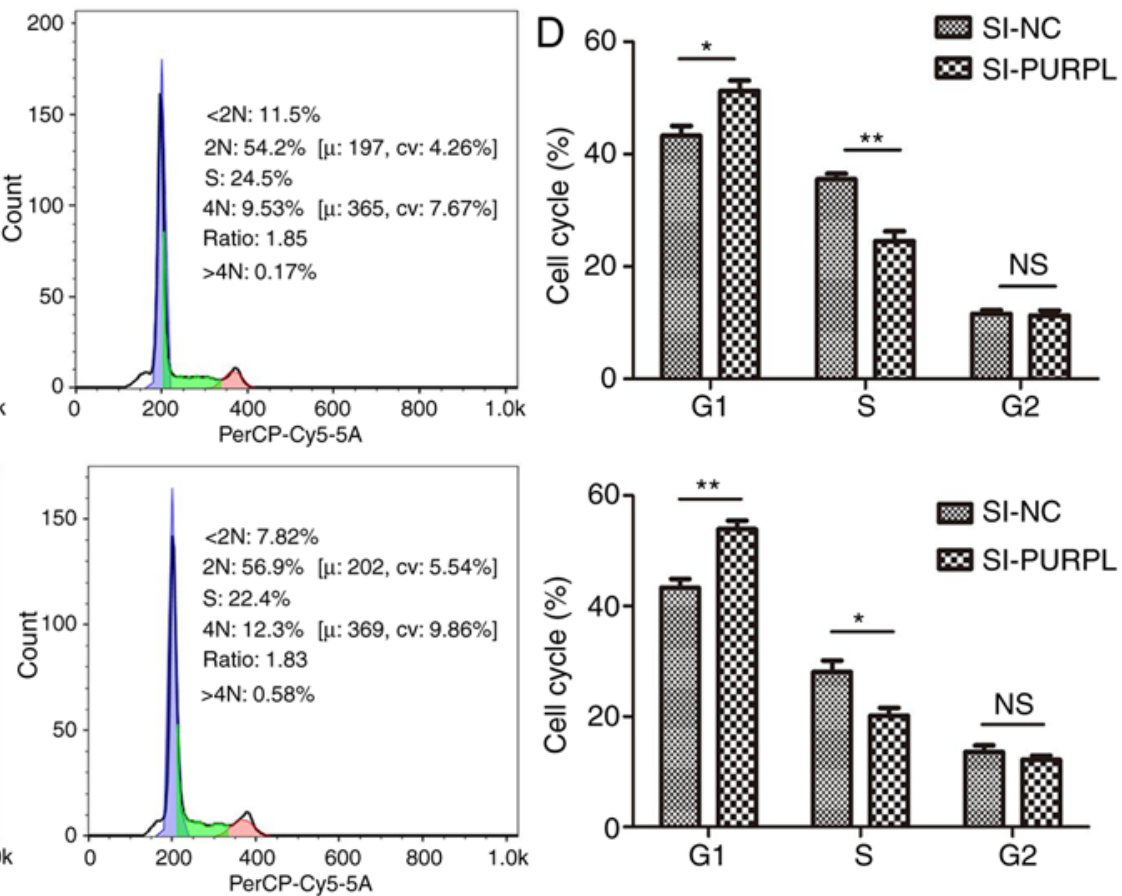

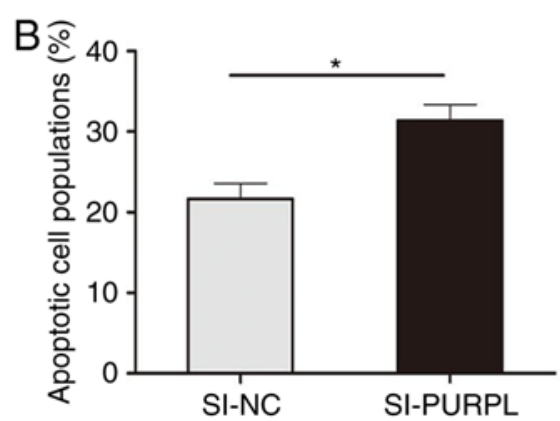

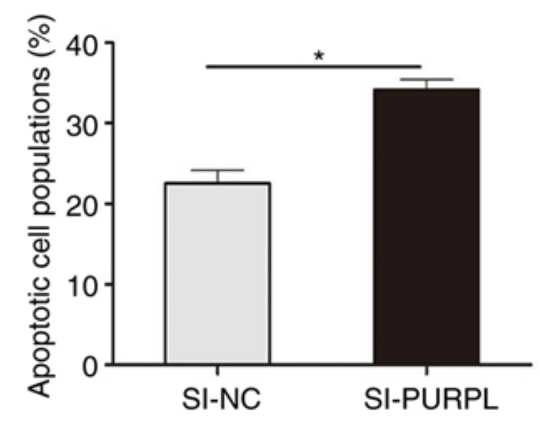

Figure 3. Long noncoding RNA-PURPL inhibits HepG2 and Huh7 apoptosis and promotes cell cycle progression. Effect of PURPL silencing on apoptosis and cell cycle progression of HepG2 and Huh7 cells, as determined by flow cytometry. (A and C) Representative flow cytometry plots and (B and D) corresponding quantification are presented. All results were analyzed using a Student's t-test. NS, not significant. ${ }^{*} \mathrm{P}<0.05$ and ${ }^{* *} \mathrm{P}<0.01$. Error bars correspond to the standard deviation. NC, negative control; PURPL, p53 upregulated regulator of P53 levels; SI, small interfering RNA.

Depletion of IncRNA PURPL promotes liver cancer cell apoptosis and leads to liver cancer cell cycle arrest. Cell apoptosis was monitored by flow cytometry. The number of apoptotic cells was significantly increased following transfection with siRNA-PURPL than those transfected with si-NC $(\mathrm{P}<0.01$; Fig. 3A and B). Regarding cell cycle progression, PURPL knockdown induced G1 phase arrest of liver cancer cells; siRNA-PURPL transfection resulted in a significant decrease in the number of cells in $\mathrm{S}$ phase compared with the control $(\mathrm{P}<0.05$; Fig. 3C and D). Collectively, these results suggested that PURPL knockdown may induce G1 phase arrest and promote liver cancer cell apoptosis.

LncRNA PURPL inhibits p53 expression in liver cancer. p53 can be modulated by lncRNAs to regulate cancer cell progression and PURPL has been reported to inactivate $\mathrm{p} 53$ protein in 
A

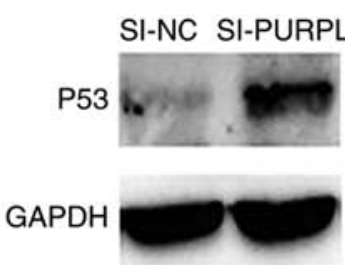

HepG2

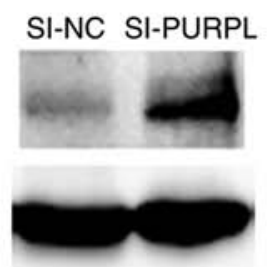

Huh7

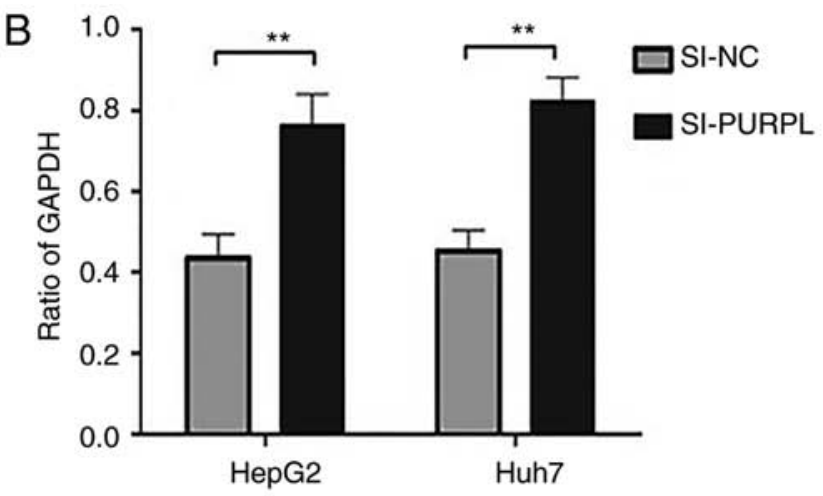

C

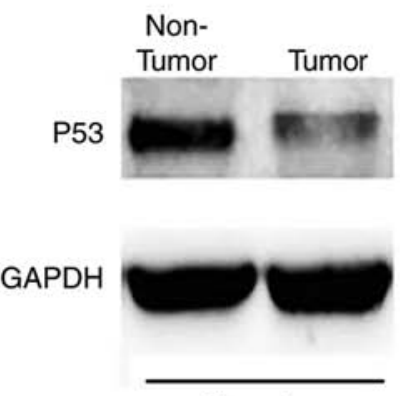

Case1

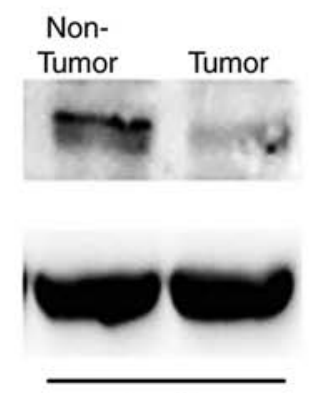

Case2

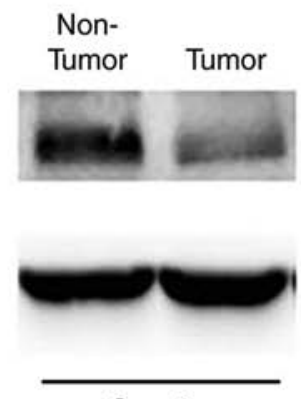

Case3
Non-
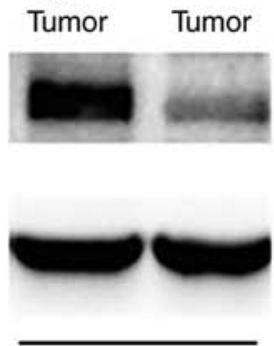

Case4

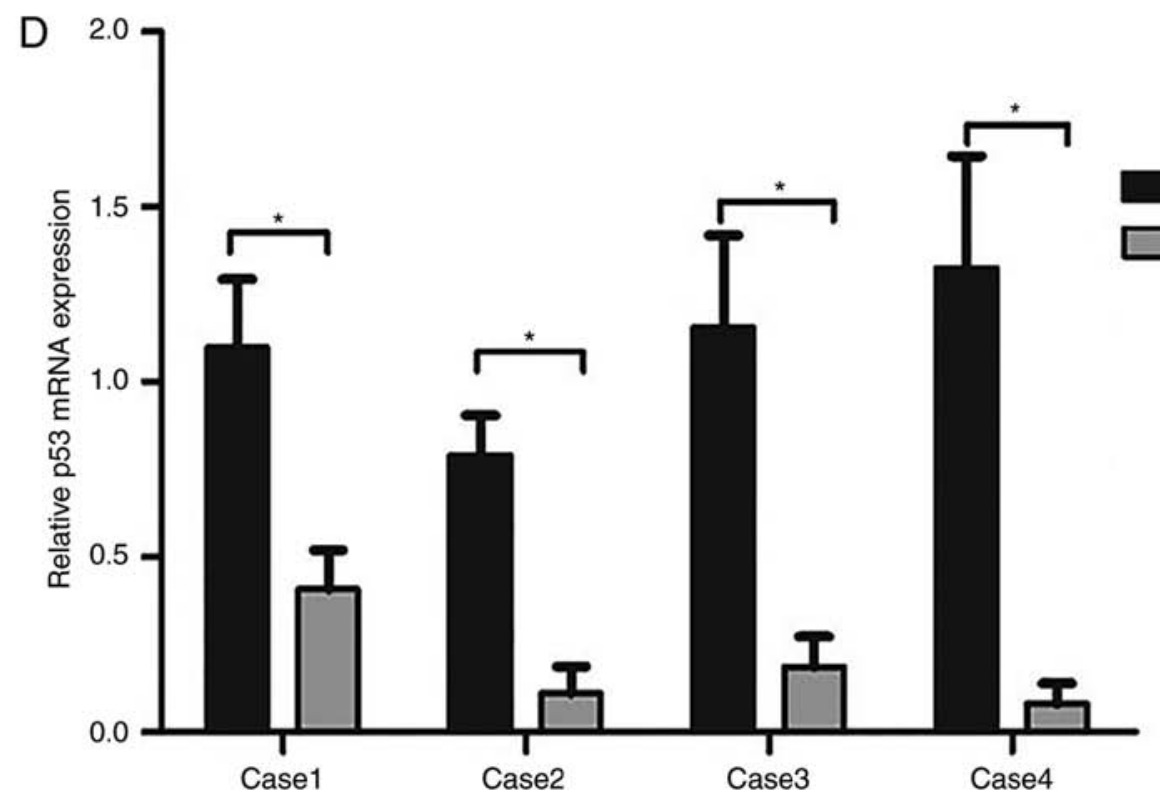

Figure 4. Long noncoding RNA-PURPL inhibits p53 expression. (A and B) Expression of p53 protein in HepG2 and Huh7 cells transfected with SI-PURPL or SI-NC. GAPDH was used as internal control. (C) p53 protein expression in liver extracts obtained from four patients with advanced liver cancer. (D) p53 mRNA expression, as determined reverse transcription-quantitative polymerase chain reaction, in liver extracts from the same four advanced liver cancer patients. All results were analyzed using a Student's t-test. ${ }^{*}<0.05$ and ${ }^{* * *} \mathrm{P}<0.01$. Error bars correspond to the standard deviation. NC, negative control; PURPL, p53 upregulated regulator of P53 levels; SI, small interfering RNA.

colorectal cancer (14). To improve understanding of the role of PURPL in liver cancer, we used western blotting to measure the expression levels of p53 in liver cancer cells. The results revealed that p53 was significantly upregulated in HepG2 and Huh7 cells transfected with siRNA-PURPL compared with si-NC $(\mathrm{P}<0.01$; Fig. 4A and B). Western blotting and RT-qPCR of liver cancer tissues demonstrated downregulation of p53 in tumor tissue compared with paracancerous tissues in four randomly selected late-stage liver cancer patients, at the protein and mRNA levels $(\mathrm{P}<0.05$; Fig. $4 \mathrm{C}$ and $\mathrm{D})$. Additionally, relative p53 mRNA expression was negatively correlated with PURPL expression in all 71 patients $(\mathrm{P}<0.001$; Fig. 5). These results indicate the role of PURPL as an oncogene that may be partially responsible for inducing p53 in liver cancer.

\section{Discussion}

Aggressive tumor invasion, metastatic dissemination, recurrence, and drug resistance are characteristic of patients with liver cancer (16-19). Thus, research on liver cancer has focused 


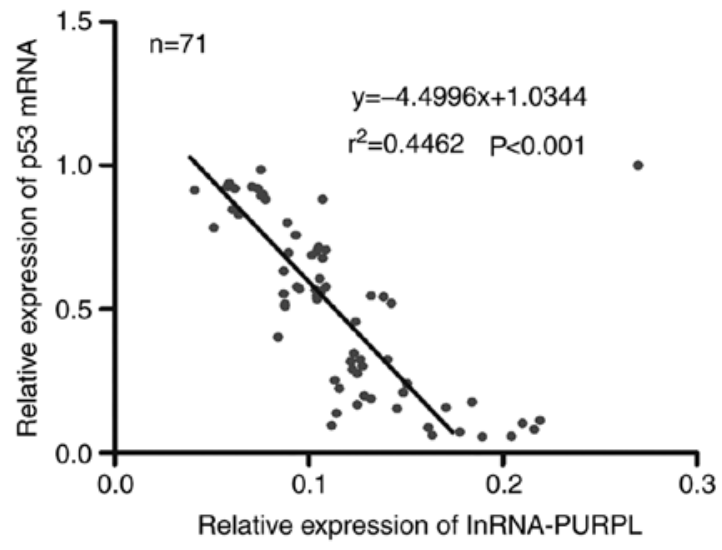

Figure 5. p53 expression is negatively correlated with PURPL expression levels. Negative correlation between relative p53 mRNA expression and PURPL mRNA expression in 71 patients with liver cancer $\left(r^{2}=0.4462\right.$, $\mathrm{P}<0.001$ ). NC, negative control; lncRNA-PURPL, long noncoding RNA p53 upregulated regulator of P53 levels.

on identifying genes that induce hepatocarcinogenesis and promote the development of liver cancer, for which targeted drugs and molecular markers capable of early diagnosis could be developed. Increasing evidence suggests that aberrant expression of lncRNA serves a key role in the development and progression of liver cancer. For example, IncRNA-CCAL promotes liver cancer progression by modulating AP-2 $\alpha$ and Wnt/ $\beta$-catenin pathways, IncRNA-HULC promotes liver cancer by increasing the expression of the high mobility group AT-hook 2 oncogene and sequestration of miRNA-186; IncRNA-TSLNC8 serves as a tumor suppressor that inactivates the interleukin-6/signal transducer and activator or transcription 3 signaling pathway in liver cancer (20-22).

LncRNA-PURPL is highly expressed in colorectal cancer and serves an important role in carcinogenesis. The present study analyzed the expression of PURPL by RT-qPCR and revealed that PURPL was highly expressed in liver cancer tissues and numerous cell lines. Furthermore, the upregulated expression of PURPL was positively associated with tumor size and tumor differentiation in patients with liver cancer. In addition, silencing of PURPL expression could inhibit liver cancer cell proliferation, arrest cell cycle progression and promote cell apoptosis.

The p53 gene was first described in 1979 (23). It serves an important role in the occurrence and development of various tumors via the control of DNA damage repair, apoptosis and cell cycle regulation $(23,24)$. Several proteins, miRNAs and IncRNAs have been reported to affect the occurrence and development of liver cancer by regulating the p53 gene (25-29).

In recent years, numerous studies have investigated on the role of lncRNAs in liver cancer and p53 gene regulation. For example, Zhou et al (30) reported that p53 regulation associated lncRNA inhibited liver cancer growth and induced apoptosis in vivo and in vitro via p53. Ren et al (31) reported that IncRNA prostate-cancer-associated ncRNA transcript-1 expression could be regulated by miR-215, a p53-inducible miRNA in liver cancer, and this post-transcriptional regulation significantly affected a variety of malignant phenomena of liver cancer cells. Additionally, Zhang et al (32) revealed that IncRNA-small nucleolar RNA host gene was a predictor of poor prognosis and promoted liver cancer tumorigenesis by regulating p53. In colorectal cancer, PURPL inhibits p53 gene expression by blocking the interaction between p53 and MYBBP1A (14). Whether PURPL inhibits the p53 gene via MYBBP1A or through other mechanisms of action requires further investigation.

In conclusion, our data demonstrated that PURPL is upregulated in liver cancer tissues, and is associated with tumor size and tumor differentiation. Silencing of PURPL could inhibit the proliferation of liver cancer cells, block cell cycle progression and promote apoptosis. Furthermore, PURPL may affect these malignant features of liver cancer cells by regulating p53. In this regard, we elevated the expression of PURPL in liver cancer cell lines, however this failed to alter the malignant characteristics of liver cancer cells (data not shown). Our future studies aim to investigate the specific mechanisms whereby PURPL affects the development of liver cancer. Collectively, lncRNA-PURPL was proposed to exert an important carcinogenic effect on liver cancer, and may be a potential predictor and a novel therapeutic target for the treatment of this disease.

\section{Acknowledgements}

Not applicable.

\section{Funding}

This study was funded by the Shenyang Municipal Science and Technology Bureau Population and Health Research Project (grant no. F15-139-9-25) and by the Natural Science Foundation of Liaoning Province (grant no. 201520529).

\section{Availability of data and materials}

The datasets used or analyzed during the current study are available from the corresponding author on reasonable request.

\section{Authors' contributions}

GW made substantial contributions to the design of the present study. XF conducted all the experiments and wrote the manuscript. YW, WZ, SX and WW made substantial contributions to the analysis of the data and discussed the results. All authors approved the final version of the manuscript.

\section{Ethics approval and consent to participate}

All patients provided written informed consent for the use of tissues. The present study was approved by the institutional ethics committee of China Medical University (Shenyang, China).

\section{Patient consent for publication}

Not applicable.

\section{Competing interests}

The authors declare that they have no competing interests. 


\section{References}

1. Shi X, Zhu HR, Liu TT, Shen XZ and Zhu JM: The Hippo pathway in hepatocellular carcinoma: Non-coding RNAs in action. Cancer Lett 400: 175-182, 2017.

2. Torre LA, Bray F, Siegel RL, Ferlay J, Lortet-Tieulent J and Jemal A: Global cancer statistics, 2012. CA Cancer J Clin 65: 87-108, 2015.

3. Fujiwara N, Friedman SL, Goossens N and Hoshida Y: Risk factors and prevention of hepatocellular carcinoma in the era of precision medicine. J Hepatol 68: 526-549, 2018.

4. Ponting CP, Oliver PL and Reik W: Evolution and functions of long noncoding RNAs. Cell 136: 629-641, 2009.

5. Ma M, Cai B, Jiang L, Abdalla BA, Li Z, Nie Q and Zhang X: lncRNA-Six1 Is a target of miR-1611 that functions as a ceRNA to regulate six 1 protein expression and fiber type switching in chicken myogenesis. Cells 7: pii: E243, 2018.

6. Grelet S, Link LA, Howley B, Obellianne C, Palanisamy V, Gangaraju VK, Diehl JA and Howe PH: A regulated PNUTS mRNA to lncRNA splice switch mediates EMT and tumour progression. Nat Cell Biol 19: 1105-1115, 2017.

7. Ferrè F, Colantoni $A$ and Helmer-Citterich M: Revealing protein-lncRNA interaction. Brief Bioinform 17: 106-116, 2016.

8. Zhu Y, Bian Y, Zhang Q, Hu J, Li L, Yang M, Qian H, Yu L, Liu B and Qian X: Construction and analysis of dysregulated IncRNA-associated ceRNA network in colorectal cancer. J Cell Biochem: Dec 7, 2018 (Epub ahead of print).

9. Jiang N, Wang X, Xie X, Liao Y, Liu N, Liu J, Miao N, Shen J and Peng T: lncRNA DANCR promotes tumor progression and cancer stemness features in osteosarcoma by upregulating AXL via miR-33a-5p inhibition. Cancer Lett 405: 46-55, 2017.

10. Li W, Jia G, Qu Y, Du Q, Liu B and Liu B: Long non-coding RNA (LncRNA) HOXA11-AS promotes breast cancer invasion and metastasis by regulating epithelial-mesenchymal transition. Med Sci Monit 23: 3393-3403, 2017.

11. Hu B, Cai H, Zheng R, Yang S, Zhou Z and Tu J: Long non-coding RNA 657 suppresses hepatocellular carcinoma cell growth by acting as a molecular sponge of miR-106a-5p to regulate PTEN expression. Int J Biochem Cell Biol 92: 34-42, 2017.

12. Mineo M, Ricklefs F, Rooj AK, Lyons SM, Ivanov P, Ansari KI, Nakano I, Chiocca EA, Godlewski J and Bronisz A: The long non-coding RNA HIF1A-AS2 facilitates the maintenance of mesenchymal glioblastoma stem-like cells in hypoxic niches. Cell Rep 15: 2500-2509, 2016.

13. Zhang HY, Zheng FS, Yang W and Lu JB: The long non-coding RNA MIAT regulates zinc finger E-box binding homeobox 1 expression by sponging miR-150 and promoteing cell invasion in non-small-cell lung cancer. Gene 633: 61-65, 2017.

14. Li XL, Subramanian M, Jones MF, Chaudhary R, Singh DK, Zong X, Gryder B, Sindri S, Mo M, Schetter A, et al: Long noncoding RNA PURPL suppresses basal p53 levels and promotes tumorigenicity in colorectal cancer. Cell Rep 20: 2408-2423, 2017.

15. Livak KJ and Schmittgen TD: Analysis of relative gene expression data using real-time quantitative PCR and the 2(-Delta Delta C(T)) method. Methods 25: 402-408, 2001.

16. Zhang T, Liu W, Meng W, Zhao H, Yang Q, Gu SJ, Xiao CC, Jia CC and Fu BS: Downregulation of miR-542-3p promotes cancer metastasis through activating TGF- $\beta /$ Smad signaling in hepatocellular carcinoma. Onco Targets Ther 11: 1929-1939, 2018.
17. Ma Y, Yang Y, Wang F, Moyer MP, Wei Q, Zhang P, Yang Z, Liu W, Zhang H, Chen N, et al: Long non-coding RNA CCAL regulates colorectal cancer progression by activating Wnt $/ \beta$-catenin signalling pathway via suppression of activator protein $2 \alpha$. Gut 65: 1494-1504, 2016

18. Wu G, Zheng K, Xia S, Wang Y, Meng X, Qin X and Cheng Y: MicroRNA-655-3p functions as a tumor suppressor by regulating ADAM10 and $\beta$-catenin pathway in Hepatocellular Carcinoma. J Exp Clin Cancer Res 35: 89, 2016.

19. Lohitesh K, Chowdhury R and Mukherjee S: Resistance a major hindrance to chemotherapy in hepatocellular carcinoma: An insight. Cancer Cell Int 18: 44, 2018.

20. Wang Y, Chen F, Zhao M, Yang Z, Li J, Zhang S, Zhang W, Ye $\mathrm{L}$ and Zhang $\mathrm{X}$ : The long noncoding RNA HULC promotes liver cancer by increasing the expression of the HMGA2 oncogene via sequestration of the microRNA-186. J Biol Chem 292: 15395-15407, 2017.

21. Zhang J: Long noncoding RNA TSLNC8 is a tumor suppressor that inactivates the interleukin-6/STAT3 signaling pathway. Hepatology 67: 171-187, 2018.

22. Liu Y, Yang Y, Wang T, Wang L, Wang X, Li T, Shi Y and Wang Y: Long non-coding RNA CCAL promotes hepatocellular carcinoma progression by regulating AP- $2 \alpha$ and $\mathrm{Wnt} / \beta$-catenin pathway. Int J Biol Macromol 109: 424-434, 2018.

23. Vogelstein B, Lane D and Levine AJ: Surfing the p53 network. Nature 408: 307-310, 2000.

24. Vousden KH and Lu X: Live or let die: The cell's response to p53. Nat Rev Cancer 2: 594-604, 2002.

25. Zhu H, Wang J, Yin J, Lu B, Yang Q, Wan Y and Jia C: Downregulation of PRAME suppresses proliferation and promotes apoptosis in hepatocellular carcinoma through the activation of P53 mediated pathway. Cell Physiol Biochem 45: 1121-1135, 2018.

26. Meng X, Franklin DA, Dong J and Zhang Y: MDM2-p53 pathway in hepatocellular carcinoma. Cancer Res 74: 7161-7167, 2014.

27. Su P, Wang F, Qi B, Wang T and Zhang S: P53 Regulationassociation long non-coding RNA (LncRNA PRAL) inhibits cell proliferation by regulation of P53 in human lung cancer. Med Sci Monit 23: 1751-1758, 2017.

28. Pollutri D, Gramantieri L, Bolondi L and Fornari F: TP53/MicroRNA interplay in hepatocellular carcinoma. Int $\mathrm{J}$ Mol Sci 17: pii: E2029, 2016.

29. Liu J, Rao J, Lou X, Zhai J, Ni Z and Wang X: Upregulated TRIM11 exerts its oncogenic effects in hepatocellular carcinoma through inhibition of P53. Cell Physiol Biochem 44: 255-266, 2017.

30. Zhou CC, Yang F, Yuan SX, Ma JZ, Liu F, Yuan JH, Bi FR, Lin KY, Yin JH, Cao GW, et al: Systemic genome screening identifies the outcome associated focal loss of long noncoding RNA PRAL in hepatocellular carcinoma. Hepatology 63: 850-863, 2016.

31. Ren Y, Shang J, Li J, Liu W, Zhang Z, Yuan J and Yang M: The long noncoding RNA PCAT-1 links the microRNA miR-215 to oncogene CRKL-mediated signaling in hepatocellular carcinoma. J Biol Chem 292: 17939-17949, 2017.

32. Zhang M, Wang W, Li T, Yu X, Zhu Y, Ding F, Li D and Yang T: Long noncoding RNA SNHG1 predicts a poor prognosis and promotes hepatocellular carcinoma tumorigenesis. Biomed Pharmacother 80: 73-79, 2016. 